\title{
An unusual case of paraneoplastic nephrotic syndrome in a patient with chronic lymphocytic leukemia
}

(1) Hematology Dept, Bordet Institute; (2) Internal Medicine Dept, Epicura Hospital, Hornu, (3) Pathology and (4) Nephrology Depts, Erasme University Hospital, Brussels, Belgium

\section{Objective}

Although frequent, chronic lymphocytic leukemia $(C L L)$ is very rarely associated with glomerular disease. We report a case of a 76-year-old man with untreated CLL for 3 years and who rapidly developed overt nephrotic syndrome (NS) and renal failure. Renal biopsy resulted in the histological diagnosis of membrano-proliferative glomerulonephritis (MPGN)

\section{Methods and Results}

Our patient's hematological malignancy was diagnosed in 2013 (13q- deletion) and did not require specific treatment until the end of 2016 . $\mathrm{He}$ presented with asthenia, dyspnea and a significant edema of the lower limbs (weight gain 5 $\mathrm{kg}$ ). Biological abnormalities were the following (Table). Abdominal ultrasonography showed 2 kidneys with normal size, without hydronephrosis and a marked splenomegaly.

Renal biopsy under ultrasound control was performed last April. Light microscopy (Figures 1a $\&$ 1b) revealed typical lesions of endocapillary proliferation with focal polymorphonuclear cell infiltration, a double contour aspect of the capillary walls and a slight increase in the mesangial matrix. Immunofluorescence stainings (Figures 2a \& 2b) were positive for IgG, C3, lambda and traces of IgM within basal membranes and mesangia deposits (no anti-C1Q or anti-IgA).

Chemotherapy was started with chlorambucil 6 $\mathrm{mg} /$ day (14 days) and methylprednisolone 32 $\mathrm{mg} /$ day, resulting in a sharp reduction of lymphocytosis. A dose of Gazyvaro $\AA$ (obinutuzumab) was then given in June 2017. As lower limb infiltration was uncontrolled with Burinex $®$ (bumetanide) alone (10 $\mathrm{mg} /$ day) continuous venovenous hemofiltration had to be performed for 4 days during a short hospital stay.

Detection of TP53 mutation in the CLL cells, by next generation sequencing allowed us to initiate a treatment by ibrutinib $(420 \mathrm{mg} /$ day). After a peak of serum creatinine reaching $3.5 \mathrm{mg} / \mathrm{dL}$, this parameter decreased to $1.6 \mathrm{mg} / \mathrm{dL}$ at the last control. There was a notable increase in the lymphocyte count as usually seen with the introduction of ibrutinib (Figure 3). The body weight is now stable but nephrotic syndrome is still present $\left(\mathrm{U}_{\text {Prot }} / \mathrm{U}_{\text {Creat }}: 3.7 \mathrm{~g} / \mathrm{g}\right.$ creat $)$

\section{Discussion}

Immune complex-mediated MPGN may be associated with progressing CLL, causing severe NS and renal failure (ref.1,2,4). According to the literature, initiation of chemotherapy is mandatory and may induce complete or partial remission of NS with improvement of renal function parameters (ref. $2,4)$.

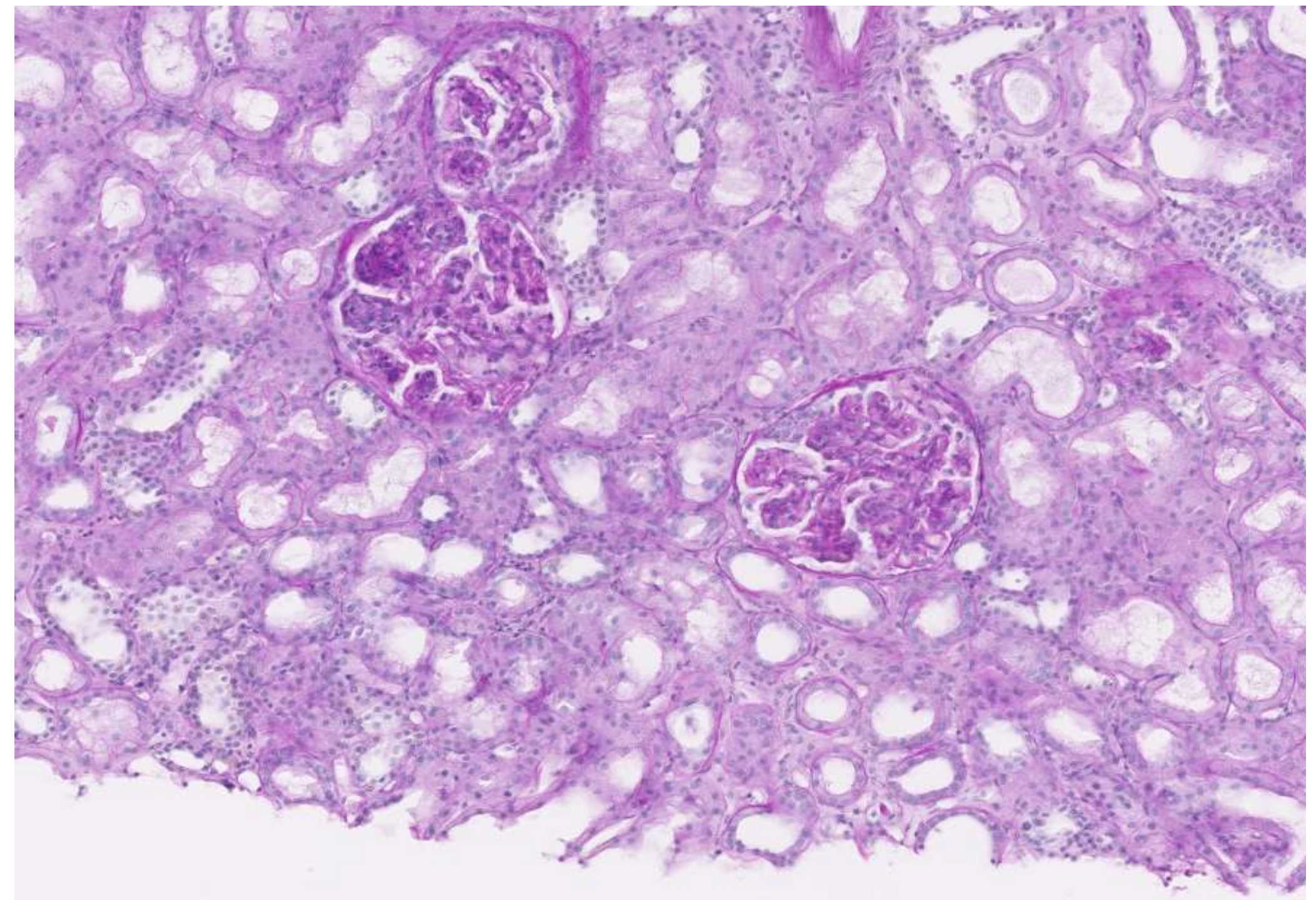

Figure 1a. Renal biopsy (light microscopy; PAS $\times 7$ )

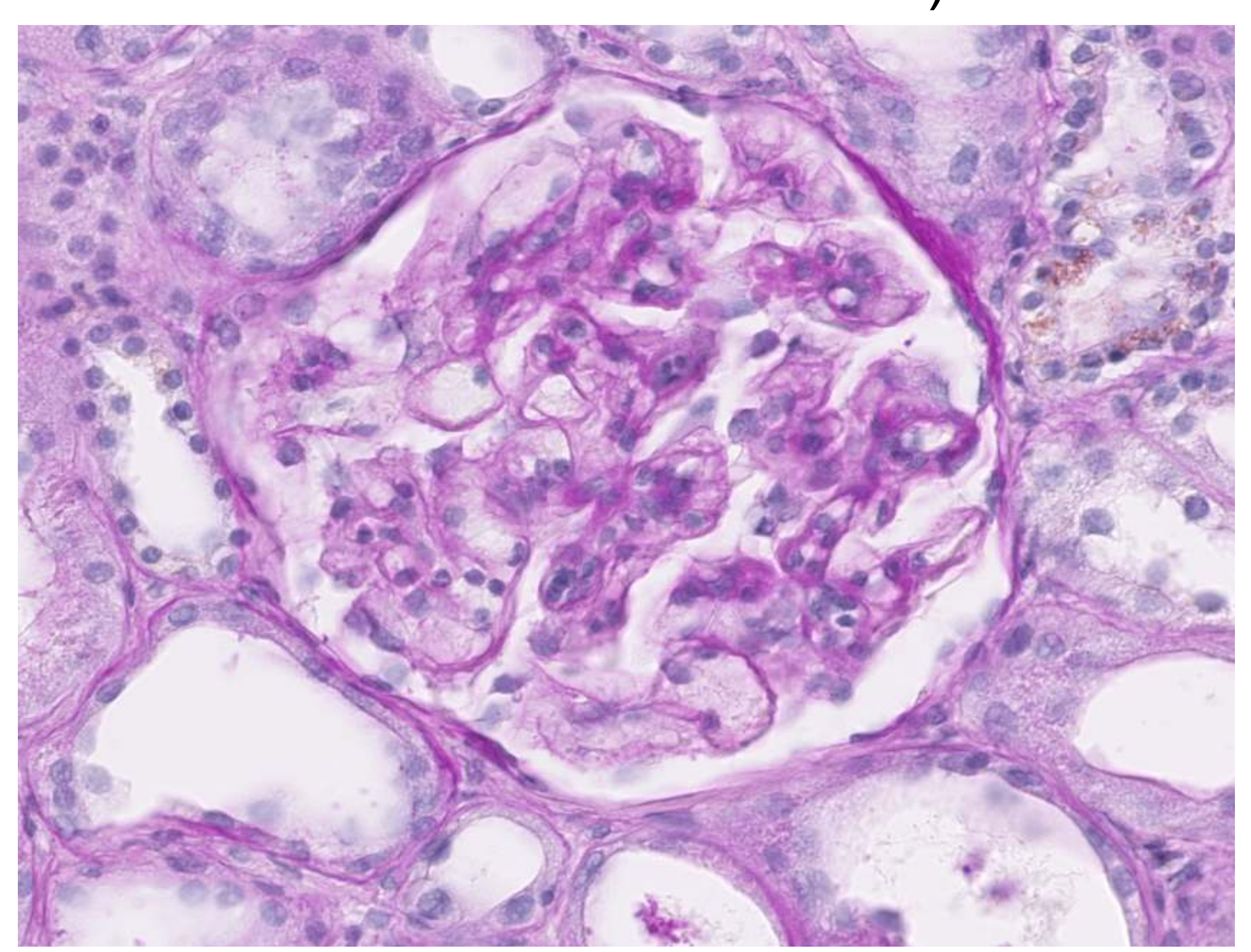

Figure 1b. Renal biopsy (light microscopy; PAS $\times 40$ )

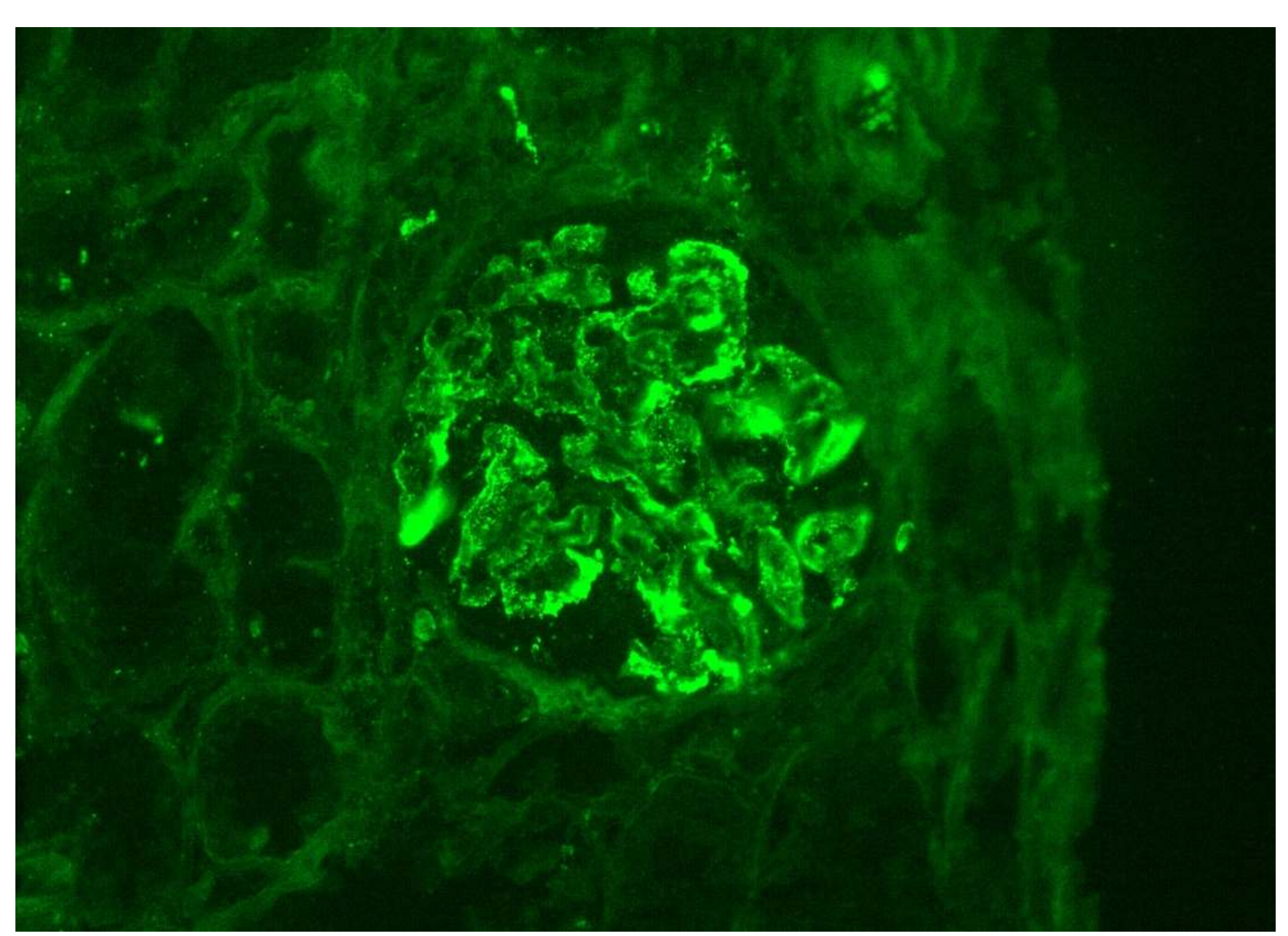

Figure 2a. Immunofluorescence IgG staining

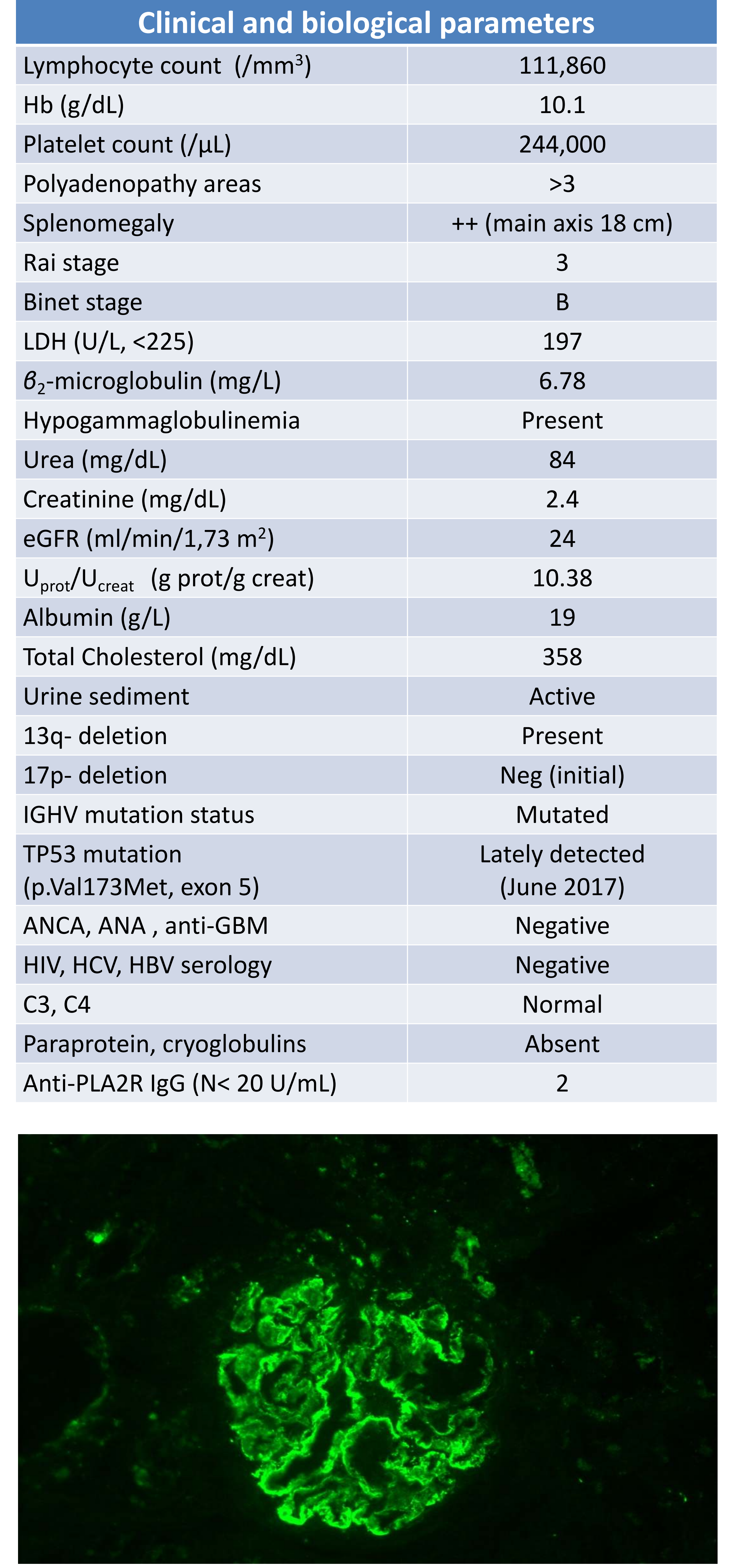

Figure 2b. Immunofluorescence $\mathrm{C}_{3}$ staining

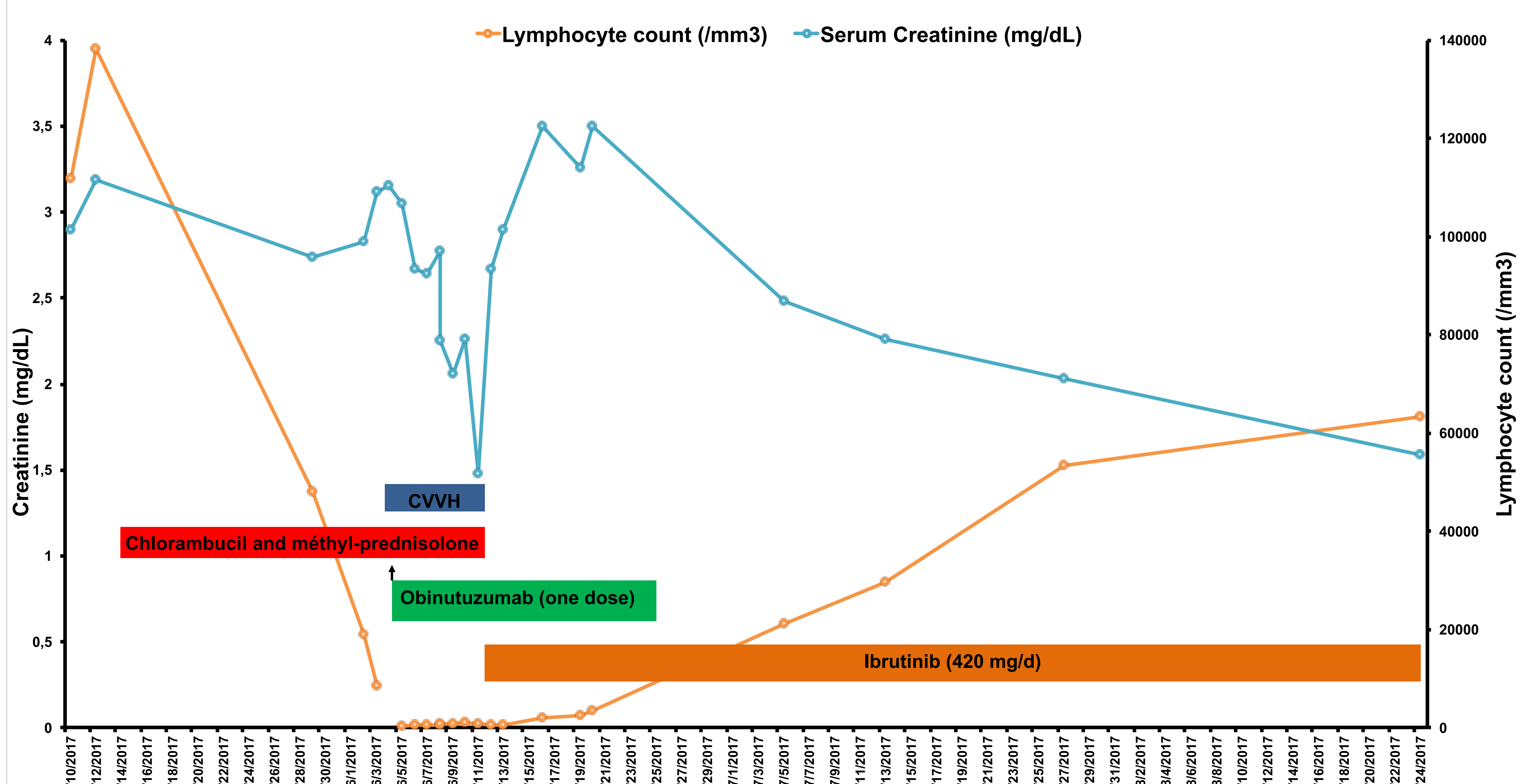

Figure 3. Time course of plasma creatinine and lymphocyte count from renal biopsy (April 2017)

\section{References}

1. Moulin B. et al. Kidney Int. 1992; 42: 127-135

2. Sethi S. et al. N Engl J Med 2012; 366: 1119-1131

3. Jain N. et al. Anderson Manual of Medical Oncology, $3^{\text {rd }}$ ed. 2016; 41-60

4. Sethi S. et al. Clin J Am Soc Nephrol 2010;5:770-782 